\begin{tabular}{|c|l|}
\hline Title & D'yakonov-Perel' spin relaxation in a bilayer with local structural inversion asymmetry \\
\hline Author(s) & Hayashida, Kenji; A kera, Hiroshi \\
\hline Citation & $\begin{array}{l}\text { Physical Review B, 101/3), 035306 } \\
\text { https://doi.org/10.1103/PhysRevB.101.035306 }\end{array}$ \\
\hline Issue Date & 2020-01-31 \\
\hline Doc URL & http://hdl.handle.net/2115/76925 \\
\hline Rights & Copyright (2020) by The A merican Physical Society. \\
\hline Type & article \\
\hline File Information & PhysRevB.101.035306.pdf \\
\hline
\end{tabular}

Instructions for use 


\title{
D'yakonov-Perel' spin relaxation in a bilayer with local structural inversion asymmetry
}

\author{
Kenji Hayashida and Hiroshi Akera $\odot$ \\ Division of Applied Physics, Graduate School and Faculty of Engineering, Hokkaido University, Sapporo, Hokkaido 060-8628, Japan
}

(Received 25 October 2019; published 31 January 2020)

\begin{abstract}
The spin relaxation in the D'yakonov-Perel' mechanism is theoretically studied in a symmetric doublequantum-well structure (DQWS) with an intersubband spin-orbit interaction (SOI) due to the local structural inversion asymmetry and the linear-in-wave-number Dresselhaus SOI. It is found that the spin relaxation rate induced by the intersubband SOI exhibits a suppression with $\omega \tau_{p}$ in the Lorentzian form of $\left(1+\omega^{2} \tau_{p}^{2}\right)^{-1}$, where $\hbar \omega$ is the intersubband energy separation and $\tau_{p}$ is the momentum relaxation time. The present Lorentzian suppression leads to a crossover with increasing $\omega \tau_{p}$ from the D'yakonov-Perel'-type relaxation (the spin relaxation time $\left.\tau_{s} \propto \tau_{p}^{-1}\right)$ to the Elliott-Yafet-type relaxation $\left(\tau_{s} \propto \tau_{p}\right)$. It is also shown that the spin relaxation rate in the presence of both the linear Dresselhaus SOI and the intersubband SOI is isotropic with respect to the in-plane spin direction in the present DQWS in contrast to a single quantum well exhibiting an in-plane anisotropy.
\end{abstract}

DOI: 10.1103/PhysRevB.101.035306

\section{INTRODUCTION}

Control of the spin relaxation [1-3] is a challenge in realizing the spintronic applications which range from the information storage to the processing [4-6]. The spin relaxation for electrons in the conduction band of nonmagnetic semiconductors has been extensively studied and is known to depend strongly on the spatial inversion symmetry of the system. In the presence of the inversion symmetry, the dominant mechanism of the spin relaxation is the Elliott-Yafet (EY) mechanism [7,8] in which the electron spin flips at a scattering from impurities and phonons through the spin-orbit interaction (SOI) induced by the scattering potential [7-10]. In the EY mechanism, the spin relaxation time $\tau_{s}$ is proportional to the momentum relaxation time $\tau_{p}\left(\tau_{s} \propto \tau_{p}\right)$. In the absence of the inversion symmetry, an additional spin relaxation is caused by the D'yakonov-Perel' (DP) mechanism [11-13] in which the effective magnetic field (EMF) created by the broken inversion symmetry induces the electron spin precession and the scattering changes the direction of the EMF. In the DP mechanism, which is stronger than the EY mechanism in most cases, the spin relaxation time is proportional to $\tau_{p}^{-1}\left(\tau_{s} \propto\right.$ $\tau_{p}^{-1}$ ). In this paper we focus on the spin relaxation caused by the locally broken inversion symmetry in a system with the global inversion symmetry and show that both the EY-type relaxation $\left(\tau_{s} \propto \tau_{p}\right)$ and the DP-type relaxation $\left(\tau_{s} \propto \tau_{p}^{-1}\right)$ appear depending on the magnitude of the effect of the locally broken inversion symmetry.

Locally broken inversion symmetry [14] is an emergent concept which has recently been addressed by many theoretical and experimental works [14-18]. A crystal with the global inversion symmetry has, at least, one inversion center. Even a crystal with the inversion center can have a pair of atomic sites, $A$ and $B$, with respect to each of which the inversion symmetry is broken so that the EMF at each site is nonzero: $\boldsymbol{B}_{\text {eff }}^{A} \neq 0$ and $\boldsymbol{B}_{\text {eff }}^{B} \neq 0$. Since $A$ and $B$ sites exchange their positions under the inversion operation with respect to one of the inversion centers, the EMFs at the two sites are antiparallel: $\boldsymbol{B}_{\text {eff }}^{A}+\boldsymbol{B}_{\text {eff }}^{B}=0$. The absence of the inversion symmetry with respect to an atomic site in a crystal with the inversion center is called locally broken inversion symmetry. This local symmetry can be thought of as more fundamental than the global symmetry since the presence of the global inversion symmetry in some crystals is determined by whether $\boldsymbol{B}_{\text {eff }}^{A}+\boldsymbol{B}_{\text {eff }}^{B}=0$ or not. Locally broken inversion symmetry has been shown to exhibit properties which have been overlooked when only the global inversion symmetry is considered. A typical example is the local current-induced effective magnetic field which has already been applied to the switching of the sublattice magnetization of the antiferromagnet [17].

Among systems with locally broken inversion symmetry, we consider a symmetric double-quantum-well structure (DQWS) [16,19-28] (illustrated in Fig. 1) in which electronic states can be controlled by changing the composition and the width of the well and barrier layers and by varying the potential with use of the applied electric field and the doping. In a single quantum well $(\mathrm{QW})$ with structural inversion asymmetry (SIA), the EMF is given by the Rashba SOI [29-32], which is induced by the asymmetric QW potential. In the symmetric DQWS with the local SIA, the EMF in both the ground subband (even parity, see Fig. 1) and the first excited subband (odd parity) vanishes because the local EMFs in two wells are opposite in direction. However, the matrix element of the DQWS-potential-induced SOI between the even-parity and the odd-parity subbands, which is called the intersubband SOI $[21,23]$, is nonzero and gives rise to various phenomena such as the bilayer intrinsic spin Hall effect [21,22,26,28]. A theoretical study on the spin relaxation in such a symmetric DQWS is highly desirable in view of extensive theoretical studies performed for the spin relaxation in single quantum wells [13,33-51]. A theory of the spin relaxation in a simple DQWS can also provide a clue to understanding the observed crossover in the oxide bilayer [52] between the EY-type and the DP-type spin relaxations with changing the separation between layers. 


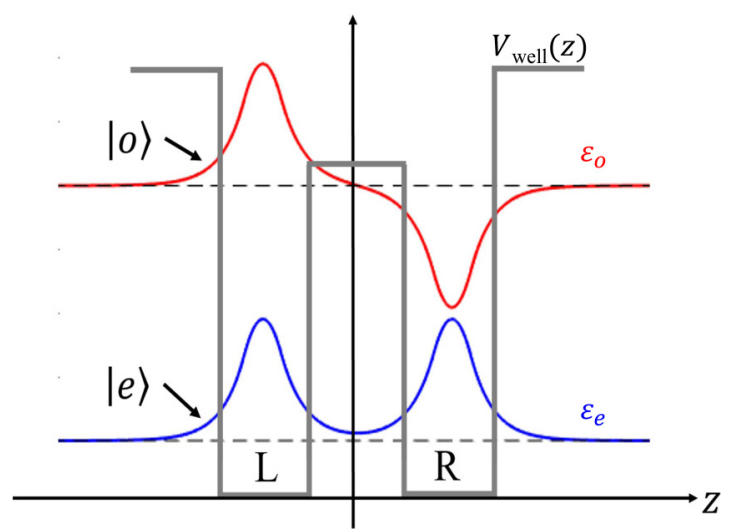

FIG. 1. Potential $V_{\text {well }}(z)$ in a DQWS and wave functions of the lowest two subbands. $|e\rangle$ is the symmetric orbital of the lowest level $\varepsilon_{e}$ (the ground subband), while $|o\rangle$ is the antisymmetric orbital of the next lowest level $\varepsilon_{o}$. These two orbitals are assumed to be a linear combination of the lowest bound states in each of the $L$ and the $R$ QWs [Eq. (14)], $|L\rangle$ and $|R\rangle$.

In this paper we theoretically study the spin relaxation in the DP mechanism in a symmetric DQWS with the local SIA (locally broken structural inversion symmetry). We take into account the ground and first excited subbands, which are described by symmetric and antisymmetric linear combinations, respectively, of localized orbitals tightly bound to each of the left and right QWs. The antiparallel EMF in the two QWs, which is induced by the local SIA, gives rise to a nonzero intersubband SOI and the vanishing intrasubband SOI (Rashba SOI). We change the magnitude of the effect of the local SIA by varying the strength of the interlayer tunneling which is described by the energy separation between the ground subband (in the energy range $\varepsilon \geqslant \varepsilon_{e}$ ) and the first excited subband $\left(\varepsilon \geqslant \varepsilon_{o}\right)$. Then we find a suppression of the spin relaxation rate with increasing the intersubband energy separation $\hbar \omega \equiv \varepsilon_{o}-\varepsilon_{e}$ in the form of the Lorentzian function $1 / \tau_{s}=\Omega_{R}^{2} \tau_{p} /\left(1+\omega^{2} \tau_{p}^{2}\right)$, with $\Omega_{R}$ being the magnitude of the Rashba EMF in each QW (in units of angular frequency) $\left(\Omega_{R}^{2} \tau_{p}\right.$ is the spin relaxation rate in a single $\mathrm{QW}$ with the Rashba EMF, $\left.\Omega_{R}\right)$. The present $\omega \tau_{p}$ dependence gives a crossover from the DP-type relaxation $\left(\tau_{s} \propto \tau_{p}^{-1}\right)$ at $\omega \tau_{p} \ll 1$ to the EY-type relaxation $\left(\tau_{s} \propto \tau_{p}\right)$ at $\omega \tau_{p} \gg 1$. We discuss the origin of this suppression of the spin relaxation due to the interlayer tunneling in the DQWS by comparing it with a similar suppression due to the cyclotron motion in a single QW [42-44,53]. (It has been shown [54] by employing a two-band model Hamiltonian that the spin relaxation rate has the form of the Lorentzian function in which $\omega \tau_{p}$ in the above expression for $1 / \tau_{s}$ is replaced with $\Delta / 2 \Gamma$, where $\Delta$ is the band gap between the two bands and $\Gamma$ is a constant imaginary part of the one-particle self-energy which has been introduced to describe the level broadening due to scatterings.)

In addition to the Rashba SOI, the Dresselhaus SOI acts on an electron in semiconductors [55] and in QWs [13] due to the bulk inversion asymmetry in zinc-blende semiconductors. In a (001)-oriented QW with both the linear-in-wave-number Dresselhaus SOI and the Rashba SOI, it has been predicted [33] and observed [56] that the spin relaxation rate depends on the direction of the spin polarization in the $\mathrm{QW}$ plane relative to the crystal axis, because the directional distribution of the EMF at the Fermi level has an in-plane anisotropy which is largest when the two SOIs are equal in strength. However we find that such anisotropy completely disappears in a symmetric DQWS with the local SIA. This can be understood by noting that the antiparallel Rashba EMF in the symmetric DQWS leads to an isotropic EMF directional distribution when averaged by the interlayer tunneling.

The outline of this paper is as follows. Section II introduces the model and the Hamiltonian of a symmetric DQWS with electrons occupying the ground and first excited subbands described by a pseudospin. Section III derives the density matrix up to first order in the SOIs. Section IV presents calculated spin relaxation rates and discussion. We demonstrate the absence of the spin relaxation anisotropy in the present DQWS as well as the Lorentzian suppression factor in the spin relaxation rate induced by the intersubband SOI. Then, we discuss the isotropy and suppression of the spin relaxation in terms of the EMF and the interlayer tunneling. Furthermore, we point out a crossover from the usual $\tau_{p}$ dependence of the DP spin relaxation rate to the anomalous EY-type dependence which occurs with increasing $\omega \tau_{p}$. Finally, Sec. V gives conclusions.

\section{HAMILTONIAN}

We consider a two-dimensional electron gas in a DQWS with electrons occupying the ground and first excited subbands. The DQWS we consider has the symmetry with respect to the center plane $(z=0)$ of the barrier between the left $(L)$ and the right $(R)$ QWs, which leads to the formation of an even-parity orbital $|e\rangle$ in the ground subband and an odd-parity orbital $|o\rangle$ in the first excited subband, while each of the two QWs has a locally broken inversion symmetry (Fig. 1). The coordinate axes $x, y$, and $z$ are oriented along [100], [010], and [001], respectively.

The Hamiltonian describing the motion of an electron in the conduction band of the DQWS is

$$
H=H_{W}+H_{D}^{\mathrm{SO}}+H_{I}^{\mathrm{SO}}+V_{\mathrm{imp}}(\hat{\boldsymbol{r}}),
$$

where

$$
\begin{array}{r}
H_{W}=\frac{\hbar^{2}}{2 m}\left(\hat{k}_{x}^{2}+\hat{k}_{y}^{2}\right)+H_{\perp}, \\
H_{\perp}=\frac{\hbar^{2}}{2 m} \hat{k}_{z}^{2}+V_{\text {well }}(\hat{z}), \\
H_{D}^{\mathrm{SO}}=-\gamma \hat{k}_{z}^{2}\left(\sigma_{x} \hat{k}_{x}-\sigma_{y} \hat{k}_{y}\right), \\
H_{I}^{\mathrm{SO}}=\eta(\hat{z})\left(\sigma_{x} \hat{k}_{y}-\sigma_{y} \hat{k}_{x}\right),
\end{array}
$$

in which $\hbar$ is the Planck constant divided by $2 \pi, m$ is the effective mass of an electron in the conduction band, $\hat{\boldsymbol{r}}=(\hat{x}, \hat{y}, \hat{z})$ is the position vector operator, $\hat{\boldsymbol{k}}=\left(\hat{k}_{x}, \hat{k}_{y}, \hat{k}_{z}\right)$ is the wave vector operator, $\boldsymbol{\sigma}=\left(\sigma_{x}, \sigma_{y}, \sigma_{z}\right)$ is the Pauli spin operator, $V_{\text {well }}$ is the potential in the DQWS due to the conduction band offset, the gate voltage, the dopants, and the electron-electron interaction in the Hartree approximation. The eigenvectors of $H_{\perp}$ are $|e\rangle$ (symmetric orbital) and $|o\rangle$ (antisymmetric orbital) and the corresponding eigenenergies are denoted by $\varepsilon_{e}$ and $\varepsilon_{o}$, respectively. $V_{\text {imp }}$ is the spin-independent potential due to randomly distributed impurities for which we employ the 
following two-dimensional model:

$$
V_{\text {imp }}(\hat{\boldsymbol{\rho}})=\sum_{i} u\left(\hat{\boldsymbol{\rho}}-\boldsymbol{\rho}_{i}\right),
$$

where $\hat{\boldsymbol{\rho}}=(\hat{x}, \hat{y}), \boldsymbol{\rho}_{i}$ represents the two-dimensional position of the $i$ th impurity, and $u$ is the potential of each impurity with cylindrical symmetry.

$H_{D}^{\text {SO }}$ is the Dresselhaus SOI linear in $\hat{k}_{x}$ and $\hat{k}_{y}$ [13] and $\gamma$ is the coefficient of the Dresselhaus SOI of constituent semiconductors [55]. The Dresselhaus SOI cubic in $\hat{k}_{x}$ and $\hat{k}_{y}$ is neglected since the expectation value of $\hat{k}_{x}^{2}$ and $\hat{k}_{y}^{2}$ is much smaller than that of $\hat{k}_{z}^{2}$ at moderate electron sheet densities [57]. $H_{I}^{\mathrm{SO}}$ is the SOI induced in the heterostructure [21,23,58], and the $z$-dependent coefficient $\eta(\hat{z})$ has contributions from the electrostatic potential and the potential due to the valence band offsets. Since both potentials are symmetric with respect to $z=0$ in the DQWS, we consider, $\eta(\hat{z})$ is an odd function of $\hat{z}$,

$$
\eta(-\hat{z})=-\eta(\hat{z}) .
$$

Thus matrix elements of $\eta(\hat{z})$ become

$$
\begin{gathered}
\langle e|\eta(\hat{z})| e\rangle=\langle o|\eta(\hat{z})| o\rangle=0, \\
\langle e|\eta(\hat{z})| o\rangle=\langle o|\eta(\hat{z})| e\rangle=\alpha_{I},
\end{gathered}
$$

which represent the intersubband SOI [21,23] induced by the local SIA, where $\alpha_{I}$ determines the strength of the intersubband SOI. We assume that state vectors with respect to the motion along the $z$ direction are described by a linear combination of $|L\rangle$ and $|R\rangle$, representing the lowest bound state in each of the $L$ and the $R \mathrm{QWs}$, so that the formulation is simplified in that some off-diagonal matrix elements between $|L\rangle$ and $|R\rangle$ vanish. In fact we can neglect off-diagonal matrix elements of the Dresselhaus SOI using this tight-binding assumption:

$$
\begin{gathered}
\left\langle L\left|\hat{k}_{z}^{2}\right| L\right\rangle=\left\langle R\left|\hat{k}_{z}^{2}\right| R\right\rangle=-\beta / \gamma, \\
\left\langle L\left|\hat{k}_{z}^{2}\right| R\right\rangle=\left\langle R\left|\hat{k}_{z}^{2}\right| L\right\rangle=0,
\end{gathered}
$$

where $\beta$ describes the strength of the Dresselhaus SOI in each of the $L$ and the $R$ QWs. We will also employ the same tightbinding assumption in Eqs. (50) and (51).

Matrix elements of $\eta(\hat{z})$ with respect to $|L\rangle$ and $|R\rangle$ are obtained to be

$$
\begin{gathered}
\langle L|\eta(\hat{z})| L\rangle=-\langle R|\eta(\hat{z})| R\rangle=\alpha_{I}, \\
\langle L|\eta(\hat{z})| R\rangle=\langle R|\eta(\hat{z})| L\rangle=0,
\end{gathered}
$$

from Eqs. (8) and (9) using the unitary transformation:

$$
|e\rangle=\frac{|L\rangle+|R\rangle}{\sqrt{2}}, \quad|o\rangle=\frac{|L\rangle-|R\rangle}{\sqrt{2}} .
$$

With use of the pseudospin operators defined by

$$
\begin{gathered}
\hat{1}_{\tau}=|L\rangle\langle L|+| R\rangle\langle R|, \\
\hat{\tau}_{X}=|L\rangle\langle L|-| R\rangle\langle R|, \\
\hat{\tau}_{Y}=i(|L\rangle\langle R|-| R\rangle\langle L|), \\
\hat{\tau}_{Z}=|L\rangle\langle R|+| R\rangle\langle L|,
\end{gathered}
$$

$H_{W}(\boldsymbol{k}) \equiv\left\langle\boldsymbol{k}\left|H_{W}\right| \boldsymbol{k}\right\rangle, \quad H_{D}^{\mathrm{SO}}(\boldsymbol{k}) \equiv\left\langle\boldsymbol{k}\left|H_{D}^{\mathrm{SO}}\right| \boldsymbol{k}\right\rangle, \quad$ and $\quad H_{I}^{\mathrm{SO}}(\boldsymbol{k}) \equiv$ $\left\langle\boldsymbol{k}\left|H_{I}^{\mathrm{SO}}\right| \boldsymbol{k}\right\rangle\left[\boldsymbol{k}=\left(k_{x}, k_{y}\right)=k(\cos \theta, \sin \theta)\right.$ with $\left.k=|\boldsymbol{k}|\right]$ are expressed as

$$
\begin{gathered}
H_{W}(\boldsymbol{k})=E_{k}-\frac{\hbar \omega}{2} \hat{\tau}_{Z}, \\
H_{D}^{\mathrm{SO}}(\boldsymbol{k})=\frac{\hbar \omega_{D}}{2} \hat{1}_{\tau}\left(\sigma_{x} \cos \theta-\sigma_{y} \sin \theta\right), \\
H_{I}^{\mathrm{SO}}(\boldsymbol{k})=\frac{\hbar \omega_{I}}{2} \hat{\tau}_{X}\left(\sigma_{x} \sin \theta-\sigma_{y} \cos \theta\right),
\end{gathered}
$$

where we have chosen $\varepsilon_{o}+\varepsilon_{e}$ as the zero of energy and

$$
\begin{gathered}
E_{k}=\hbar^{2} k^{2} / 2 m, \\
\hbar \omega=\varepsilon_{o}-\varepsilon_{e}, \\
\hbar \omega_{D}=2 \beta k, \\
\hbar \omega_{I}=2 \alpha_{I} k .
\end{gathered}
$$

\section{DENSITY MATRIX}

To obtain the spin relaxation rate, we start with the quantum Liouville equation for the density operator $\rho(t)$ describing noninteracting electrons [59],

$$
\frac{\partial \rho(t)}{\partial t}=\frac{1}{i \hbar}[H, \rho],
$$

and separate the Hamiltonian $H$ into the unperturbed Hamiltonian $H_{0}$ and the perturbation $V$,

$$
H=H_{0}+V, \quad H_{0}=H_{W}+H_{D}^{\mathrm{SO}}+H_{I}^{\mathrm{SO}}, \quad V=V_{\text {imp }} .
$$

By rewriting $[V, \rho]$ with the use of the interaction picture, we have

$$
\frac{\partial \rho(t)}{\partial t}=\frac{1}{i \hbar}\left[H_{0}, \rho\right]+\hat{\mathbf{J}} \rho,
$$

with

$$
\begin{gathered}
\hat{\mathrm{J}} \rho=e^{-i H_{0} t / \hbar} \frac{1}{i \hbar}\left[V_{I}, \rho_{I}\right] e^{i H_{0} t / \hbar}, \\
\rho_{I}(t)=\rho_{I}(0)+\frac{1}{i \hbar} \int_{0}^{t} d s\left[V_{I}(s), \rho_{I}(s)\right],
\end{gathered}
$$

where $A_{I}=e^{i H_{0} t / \hbar} A e^{-i H_{0} t / \hbar}$. We employ the lowest-order perturbation approximation with respect to $V_{\text {imp }}$ by assuming that the impurity potential is smaller than the Fermi energy. Up to second order in the perturbation $V$, which we switch on at $t=0$, we have

$$
\begin{gathered}
\hat{\mathbf{J}} \rho=(\hat{\mathrm{J}} \rho)_{1}+(\hat{\mathrm{J}} \rho)_{2}, \\
(\hat{\mathrm{J}} \rho)_{1}=e^{-i H_{0} t / \hbar} \frac{1}{i \hbar}\left[V_{I}(t), \overline{\rho_{I}(t)}\right] e^{i H_{0} t / \hbar} \\
(\hat{\mathrm{J}} \rho)_{2}=\left(\frac{1}{i \hbar}\right)^{2} \int_{0}^{t} d s e^{-i H_{0} t / \hbar}\left\{V_{I}(t),\left[V_{I}(s), \overline{\rho_{I}(t)}\right]\right\} e^{i H_{0} t / \hbar} .
\end{gathered}
$$

Here we have introduced the average of an operator $A$ with respect to the in-plane impurity configuration, which is defined 
by [59]

$$
\bar{A}=\frac{1}{S} \int_{S} \cdots \frac{1}{S} \int_{S} A d \rho_{1} \cdots d \rho_{N_{\mathrm{imp}}},
$$

where $N_{\text {imp }}$ is the number of impurities in the DQWS with the area $S$. In deriving Eqs. (32) and (33) we have used $\rho_{I}(0)=$ $\overline{\rho_{I}(t)}+O(V)$ which is obtained from Eq. (30) by using the independence of $\rho_{I}(0)$ on the impurity configuration.

In the following calculation we focus on the DP mechanism [11-13] and neglect $H_{D}^{\mathrm{SO}}$ and $H_{I}^{\mathrm{SO}}$ in $e^{i H_{0} t / \hbar}$ and $e^{-i H_{0} t / \hbar}$ in calculating $\hat{\mathrm{J}} \rho$. We calculate diagonal-in- $\boldsymbol{k}$ matrix elements, $\langle\boldsymbol{k}|\cdots| \boldsymbol{k}\rangle$, of both sides of Eq. (28) and take the average with respect to the impurity configuration. By using $\left\langle\boldsymbol{k} \mid \overline{\left(\hat{J}_{\rho} \rho\right)_{1} \mid \boldsymbol{k}}\right\rangle=$ 0 [60] we obtain the following equation for $\bar{\rho}(\boldsymbol{k}) \equiv\langle\boldsymbol{k}|\rho(t)| \boldsymbol{k}\rangle$ :

$$
\frac{\partial \bar{\rho}(\boldsymbol{k})}{\partial t}=\frac{1}{i \hbar}\left[\left\langle\boldsymbol{k}\left|H_{0}\right| \boldsymbol{k}\right\rangle, \bar{\rho}(\boldsymbol{k})\right]+\left\langle\boldsymbol{k}\left|\overline{(\hat{\mathbf{J}} \rho)_{2}}\right| \boldsymbol{k}\right\rangle
$$

where the last term, $\left\langle\boldsymbol{k}\left|\overline{(\hat{\mathbf{J}} \rho)_{2}}\right| \boldsymbol{k}\right\rangle$, becomes the collision term which includes the momentum relaxation time $\tau_{p}$ defined by

$$
\frac{1}{\tau_{p}}=\sum_{\boldsymbol{k}^{\prime}} \frac{2 \pi}{\hbar} \overline{\left|V_{\boldsymbol{k}^{\prime} \boldsymbol{k}}\right|^{2}} \delta\left(E_{k^{\prime}}-E_{k}\right)(1-\cos \phi),
$$

with $\phi$ being the angle of $\boldsymbol{k}^{\prime}$ relative to that of $\boldsymbol{k}$.

We consider the strong scattering regime [11-13], in which $\omega_{D} \tau_{p} \ll 1$ and $\omega_{I} \tau_{p} \ll 1$. Then we expand $\bar{\rho}(\boldsymbol{k})$ with respect to $\omega_{D} \tau_{p}$ and $\omega_{I} \tau_{p}$ and retain terms up to the first order:

$$
\bar{\rho}(\boldsymbol{k}) \simeq \bar{\rho}^{(0)}(\boldsymbol{k})+\bar{\rho}^{(1)}(\boldsymbol{k})
$$

The zeroth-order term $\bar{\rho}^{(0)}(\boldsymbol{k})$, which gives nonzero spin polarization, is expressed by [11-13]

$$
\bar{\rho}^{(0)}(\boldsymbol{k})=F_{00}(k)+F_{10}(k) \sigma_{s}+F_{01}(k) \hat{\tau}_{Z}+F_{11}(k) \sigma_{s} \hat{\tau}_{Z},
$$

with

$$
F_{s t}(k) \equiv \frac{1}{4} \sum_{\sigma= \pm 1} \sum_{\tau= \pm 1} \sigma^{s} \tau^{t} f_{0}\left(E_{k}-\tau \frac{\hbar \omega}{2}-\mu_{\sigma}\right),
$$

and

$$
f_{0}(\varepsilon) \equiv\left[1+\exp \left(\frac{\varepsilon}{k_{B} T}\right)\right]^{-1},
$$

where $\sigma_{s}=\boldsymbol{\sigma} \cdot \boldsymbol{e}_{s}$, with $\boldsymbol{e}_{s}$ being the unit vector in the direction of the spin polarization; $\sigma$ and $\tau$ are eigenvalues of $\sigma_{s}$ and $\hat{\tau}_{Z}$, respectively; $\mu_{\sigma}$ is the spin-dependent chemical potential; $T$ is the temperature; and $k_{B}$ is the Boltzmann constant.

By solving Eq. (35) we obtain the first-order term divided into two terms:

$$
\bar{\rho}^{(1)}(\boldsymbol{k})=\bar{\rho}_{D}^{(1)}(\boldsymbol{k})+\bar{\rho}_{I}^{(1)}(\boldsymbol{k}) .
$$

The first term comes from the Dresselhaus SOI and is given by

$$
\bar{\rho}_{D}^{(1)}(\boldsymbol{k})=\frac{\tau_{p}}{i \hbar}\left[H_{D}^{\mathrm{SO}}(\boldsymbol{k}), \bar{\rho}^{(0)}(\boldsymbol{k})\right] .
$$

The second term originates from the intersubband SOI and is expressed by

$$
\bar{\rho}_{I}^{(1)}(\boldsymbol{k})=\bar{\rho}_{I X}^{(1)}(\boldsymbol{k}) \hat{\tau}_{X}+\bar{\rho}_{I Y}^{(1)}(\boldsymbol{k}) \hat{\tau}_{Y},
$$

with

$$
\begin{aligned}
\left(\begin{array}{l}
\bar{\rho}_{I X}^{(1)}(\boldsymbol{k}) \\
\bar{\rho}_{I Y}^{(1)}(\boldsymbol{k})
\end{array}\right)= & -\frac{\omega_{I} \tau_{p}}{2\left(1+\omega^{2} \tau_{p}^{2}\right)}\left(\begin{array}{cc}
1 & \omega \tau_{p} \\
-\omega \tau_{p} & 1
\end{array}\right) \\
& \times\left(\begin{array}{c}
i F_{10}\left[\sigma_{I}, \sigma_{s}\right] \\
2 F_{01} \sigma_{I}+F_{11}\left\{\sigma_{I}, \sigma_{s}\right\}
\end{array}\right),
\end{aligned}
$$

where $\sigma_{I}=\sigma_{x} \sin \theta-\sigma_{y} \cos \theta$ and $\{A, B\}=A B+B A$.

\section{SPIN RELAXATION RATE AND DISCUSSION}

The spin relaxation rate is defined by

$$
\frac{1}{\tau_{i j}}=-\frac{d S_{i}}{d t} \frac{1}{S_{j}}, \quad i, j=x, y, z
$$

with the use of the spin polarization

$$
S_{i}=\operatorname{tr}_{\sigma, \tau}\left(\sigma_{i} \otimes 1_{\tau} \sum_{k} \bar{\rho}(\boldsymbol{k})\right)
$$

and its time derivative, where $\operatorname{tr}_{\sigma, \tau}$ is the trace operation with respect to the spin and pseudospin (layer) degrees of freedom. By substituting $\bar{\rho}^{(0)}(\boldsymbol{k})$ in Eq. (38) into the expression of $S_{j}$ and $\partial \bar{\rho}(\boldsymbol{k}) / \partial t$ in Eq. (35) with $\bar{\rho}^{(1)}(\boldsymbol{k})$ in Eq. (41) into that of $d S_{i} / d t$, we obtain

$$
\frac{2}{\tau_{x x}}=\frac{2}{\tau_{y y}}=\frac{1}{\tau_{z z}}, \quad \frac{1}{\tau_{i j}}=0(i \neq j)
$$

with

$$
\frac{1}{\tau_{z z}}=\left[\int d E_{k} F_{10}(k)\right]^{-1} \int d E_{k}\left(\omega_{D}^{2}+\frac{\omega_{I}^{2}}{1+\omega^{2} \tau_{p}^{2}}\right) \tau_{p} F_{10}(k) .
$$

The above spin relaxation rate is derived using the twodimensional impurity potential, Eq. (6). In the case of the three-dimensional impurity potential, we obtain a similar result of the spin relaxation rate with $\tau_{p} \tau_{l}$ instead of $\tau_{p}^{2}$ in Eq. (48), where $\tau_{l}$ is the lifetime defined by a formula without the factor $1-\cos \phi$ in the formula for the momentum relaxation time $\tau_{p}$, if we make the following two assumptions [60].

(i) The potential range of each impurity is short compared to the separation between two QWs. More precisely, the spherically symmetric potential of each impurity $v$, which appears in the expression of the impurity potential

$$
V_{\mathrm{imp}}(\hat{\boldsymbol{r}})=\sum_{i} v\left(\hat{\boldsymbol{r}}-\boldsymbol{r}_{i}\right)
$$

satisfies

$$
\begin{gathered}
\left\langle L\left|v\left(\hat{\boldsymbol{r}}-\boldsymbol{r}_{i}\right)\right| R\right\rangle=0, \\
\left\langle L\left|v\left(\hat{\boldsymbol{r}}-\boldsymbol{r}_{i}\right)\right| L\right\rangle\left\langle R\left|v\left(\hat{\boldsymbol{r}}-\boldsymbol{r}_{i}\right)\right| R\right\rangle=0,
\end{gathered}
$$

for each impurity.

(ii) In addition we neglect $H_{\perp}$ in $e^{i H_{0} t / \hbar}$ and $e^{-i H_{0} t / \hbar}$ in calculating $\hat{\mathrm{J}} \rho$. 
(a) L layer

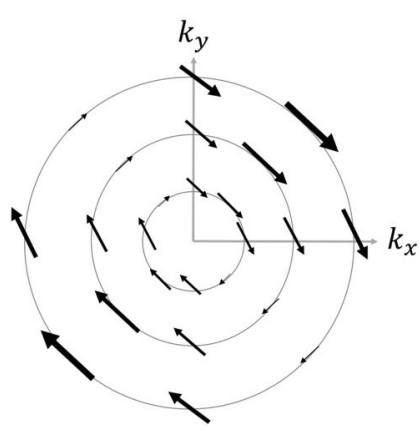

(b) R layer

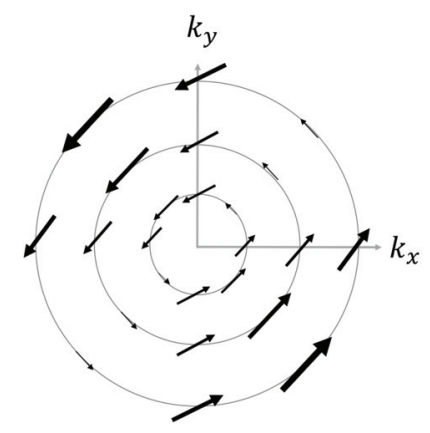

FIG. 2. Total effective magnetic field (EMF) consisting of the Dresselhaus and the intersubband SOIs in (a) the $L$ layer and (b) the $R$ layer. Each vector represents the EMF at each point in $\left(k_{x}, k_{y}\right)$ space. The $x$ and $y$ axes are taken along the [100] and [010] directions, respectively.

The derived spin relaxation rate Eq. (48) is determined by $\omega_{D}^{2} \tau_{p}, \omega_{I}^{2} \tau_{p}$, and $\omega \tau_{p}$ at the Fermi level when $k_{B} T \ll \varepsilon_{F}$ and $\hbar \omega \ll \varepsilon_{F}\left(\varepsilon_{F}\right.$ : the Fermi energy). The contribution of the Rashba term relative to that of the Dresselhaus term, $\omega_{I} / \omega_{D}=$ $\alpha_{I} / \beta$, can be changed in the range of $0<\alpha_{I} / \beta<10$ [50,61]. The dimensionless parameter $\omega \tau_{p}$ can also be changed in the range of $0<\omega \tau_{p}<10$ since $0<\hbar \omega<10 \mathrm{meV}$ and $\tau_{p} \sim$ 1 ps.

The spin relaxation rate in our symmetric DQWS exhibits two distinct features. First, the spin relaxation rate has inplane isotropy: $\tau_{x x}^{-1}=\tau_{y y}^{-1}$ and $\tau_{x y}^{-1}=\tau_{y x}^{-1}=0$ [Eq. (47)]; and second, the spin relaxation rate due to the intersubband SOI is suppressed with increasing the intersubband energy separation $\hbar \omega$ in the form of the Lorentzian function [Eq. (48)].

The inplane anisotropy in a (001)-oriented single QW with both the linear-in- $\boldsymbol{k}$ Dresselhaus SOI and the Rashba SOI [33] appears due to the cross term between the two SOIs. In our symmetric DQWS, the corresponding cross term between the Dresselhaus SOI and the intersubband SOI vanishes because of the different symmetry of the two SOIs in pseudospin space. In fact cross terms appearing in $d S_{i} / d t$,

$$
\begin{aligned}
& {\left[H_{D}^{\mathrm{SO}}(\boldsymbol{k}), \bar{\rho}_{I}^{(1)}(\boldsymbol{k})\right]=A_{X} \hat{\tau}_{X}+A_{Y} \hat{\tau}_{Y},} \\
& {\left[H_{I}^{\mathrm{SO}}(\boldsymbol{k}), \bar{\rho}_{D}^{(1)}(\boldsymbol{k})\right]=B_{X} \hat{\tau}_{X}+B_{Y} \hat{\tau}_{Y},}
\end{aligned}
$$

with $A_{X}, A_{Y}, B_{X}$, and $B_{Y}$ operators in spin space, disappear in the trace operation in pseudospin space.

The isotropy in the spin relaxation can also be understood from the angular distribution of the total EMF in each of the $L$ and the $R$ layers. Figure 2 schematically presents the sum of the EMFs induced by the linear Dresselhaus and the intersubband SOI in (a) the $L$ layer and (b) the $R$ layer. Although the EMF in each layer has an anisotropic angular distribution, the EMF which acts on electrons in the two layers has an isotropic distribution, thus leading to the isotropic spin relaxation.

The suppression of the spin relaxation rate in our symmetric DQWS [Eq. (48)] with $\omega$, which represents the angular frequency of the pseudospin precession, has the same form as that in a single $\mathrm{QW}$ with the cyclotron frequency $\omega_{c}$ in a perpendicular magnetic field [42-44,53], in which the spin relaxation rate is suppressed by $\left(1+\omega_{c}^{2} \tau_{p}^{2}\right)^{-1}$. Since the cyclotron motion changes the direction of the $\boldsymbol{k}$-dependent EMF and that of the spin precession axis, the precessioninduced spin relaxation is suppressed. In a symmetric DQWS with the antiparallel EMF, the pseudospin precession changes the direction of the layer-dependent EMF, leading to the suppression of the DP spin relaxation. It has been shown [60] that the same suppression factor, $\left(1+\omega^{2} \tau_{p}^{2}\right)^{-1}$, due to the pseudospin precession also appears in spin Hall conductivity in a DQWS.

The suppression factor $\left(1+\omega^{2} \tau_{p}^{2}\right)^{-1}$ gives a crossover from the DP-type relaxation $\left(\tau_{s} \propto \tau_{p}^{-1}\right)$ at $\omega \tau_{p} \ll 1$ to the EY-type relaxation $\left(\tau_{s} \propto \tau_{p}\right)$ at $\omega \tau_{p} \gg 1$ by considering the DP mechanism alone. A similar crossover was already derived from a two-band model Hamiltonian and a constant imaginary part of the one-particle self-energy which was introduced to describe the level broadening due to scatterings [54] as mentioned in Sec. I. The derived crossover, however, occurs around $\Delta / 2 \Gamma=1$, which corresponds to $\omega \tau_{l}=1$ with use of the lifetime $\tau_{l}$ defined by

$$
\frac{1}{\tau_{l}}=\sum_{\boldsymbol{k}^{\prime}} \frac{2 \pi}{\hbar} \overline{\left|V_{\boldsymbol{k}^{\prime} \boldsymbol{k}}\right|^{2}} \delta\left(E_{k^{\prime}}-E_{k}\right)
$$

since the band gap $\Delta$ corresponds to $\hbar \omega$ and the level broadening $2 \Gamma$ corresponds to $\hbar \tau_{l}^{-1}$, In our calculation we have exactly solved the kinetic equation with the collision term and obtained the crossover around $\omega \tau_{p}=1$. The difference between $\omega \tau_{p}=1$ and $\omega \tau_{l}=1$ is important in our system since the dominant impurity in modulation-doped QW structures has a long-range potential leading to $\tau_{p} \gg$ $\tau_{l}$ (see, for example, observed values of $\tau_{p}$ and $\tau_{l}$ in Ref. [62]).

\section{CONCLUSIONS}

We have theoretically studied the spin relaxation in the DP mechanism in a symmetric DQWS with the intersubband SOI due to the local SIA and the linear-in- $\boldsymbol{k}$ Dresselhaus SOI. We have found that the spin relaxation rate induced by the intersubband SOI exhibits a suppression with the pseudospin precession frequency in the Lorentzian form $\left(1+\omega^{2} \tau_{p}^{2}\right)^{-1}$, which is analogous to the suppression with the cyclotron frequency in a single QW [42-44,53]. The present Lorentzian suppression leads to a crossover with increasing $\omega \tau_{p}$ from the DP-type relaxation $\left(\tau_{s} \propto \tau_{p}^{-1}\right)$ to the EY-type relaxation $\left(\tau_{s} \propto \tau_{p}\right)$. We have also shown that the spin relaxation rate in the presence of both the linear Dresselhaus SOI and the intersubband SOI is isotropic with respect to the in-plane spin direction in our symmetric DQWS in contrast to a single QW exhibiting the in-plane anisotropy $[33,56]$.

The interplay in the spin relaxation between the spin and the pseudospin, which we have found in a DQWS, is also expected to appear in other electronic systems with the pseudospin degree of freedom. In graphene and bilayer graphene, which are typical systems with pseudospin, the spin relaxation 
has been extensively studied [63-67] and both the DP-type and the EY-type relaxation have been observed $[63,66,67]$. In understanding the spin dynamics including the spin relaxation in graphene and transition metal dichalcogenides, the viewpoint of the spin-pseudospin interaction will be useful.

\section{ACKNOWLEDGMENT}

This work was supported by a Grant-in-Aid for Scientific Research (C) (Grant No. JP17K05484) from the Japan Society for the Promotion of Science (JSPS).
[1] I. Žutić, J. Fabian, and S. D. Sarma, Rev. Mod. Phys. 76, 323 (2004).

[2] R. T. Harley, in Spin Physics in Semiconductors, edited by M. I. Dyakonov (Springer, Berlin, 2008), pp. 29-54.

[3] M. W. Wu, J. H. Jiang, and M. Q. Weng, Phys. Rep. 493, 61 (2010).

[4] S. Datta and B. Das, Appl. Phys. Lett. 56, 665 (1990).

[5] K. C. Hall, W. H. Lau, K. Gündoğdu, M. E. Flatté, and T. F. Boggess, Appl. Phys. Lett. 83, 2937 (2003).

[6] K. C. Hall and M. E. Flatté, Appl. Phys. Lett. 88, 162503 (2006).

[7] R. J. Elliott, Phys. Rev. 96, 266 (1954).

[8] Y. Yafet, in Solid State Physics, edited by F. Seitz and D. Turnbull (Academic, New York, 1963), Vol. 14, pp. 1-98.

[9] J. N. Chazalviel, Phys. Rev. B 11, 1555 (1975).

[10] J. Fabian and S. Das Sarma, Phys. Rev. Lett. 83, 1211 (1999).

[11] M. I. Dyakonov and V. I. Perel, Sov. Phys. JETP 33, 1053 (1971).

[12] M. I. Dyakonov and V. I. Perel, Sov. Phys. Solid State 13, 3023 (1972).

[13] M. I. Dyakonov and V. Y. Kachorovskii, Sov. Phys. Semicond. 20, 110 (1986).

[14] X. Zhang, Q. Liu, J.-W. Luo, A. J. Freeman, and A. Zunger, Nat. Phys. 10, 387 (2014).

[15] D. Maruyama, M. Sigrist, and Y. Yanase, J. Phys. Soc. Jpn. 81, 034702 (2012).

[16] S. Nakosai, Y. Tanaka, and N. Nagaosa, Phys. Rev. Lett. 108, 147003 (2012).

[17] J. Železný, H. Gao, K. Výborný, J. Zemen, J. Mašek, A. Manchon, J. Wunderlich, J. Sinova, and T. Jungwirth, Phys. Rev. Lett. 113, 157201 (2014).

[18] J. H. Ryoo and C.-H. Park, NPG Asia Mater. 9, e382 (2017).

[19] O. E. Raichev and P. Debray, Phys. Rev. B 67, 155304 (2003).

[20] V. A. Zyuzin, E. G. Mishchenko, and M. E. Raikh, Phys. Rev. B 74, 205322 (2006).

[21] E. Bernardes, J. Schliemann, M. Lee, J. C. Egues, and D. Loss, Phys. Rev. Lett. 99, 076603 (2007).

[22] P.-Q. Jin and Y.-Q. Li, Phys. Rev. B 76, 235311 (2007).

[23] R. S. Calsaverini, E. Bernardes, J. C. Egues, and D. Loss, Phys. Rev. B 78, 155313 (2008).

[24] I. V. Rozhansky and N. S. Averkiev, Phys. Rev. B 77, 115309 (2008).

[25] M. Akabori, S. Hidaka, H. Iwase, S. Yamada, and U. Ekenberg, J. Appl. Phys. 112, 113711 (2012).

[26] F. G. G. Hernandez, L. M. Nunes, G. M. Gusev, and A. K. Bakarov, Phys. Rev. B 88, 161305(R) (2013).

[27] S. Souma, A. Sawada, H. Chen, Y. Sekine, M. Eto, and T. Koga, Phys. Rev. Appl. 4, 034010 (2015).
[28] A. Khaetskii and J. C. Egues, Europhys. Lett. 118, 57006 (2017).

[29] E. I. Rashba, Sov. Phys. Solid State 2, 1109 (1960).

[30] F. J. Ohkawa and Y. Uemura, J. Phys. Soc. Jpn. 37, 1325 (1974).

[31] Y. A. Bychkov and E. I. Rashba, J. Phys. C 17, 6039 (1984).

[32] Y. A. Bychkov and E. I. Rashba, JETP Lett. 39, 78 (1984).

[33] N. S. Averkiev and L. E. Golub, Phys. Rev. B 60, 15582 (1999).

[34] A. A. Kiselev and K. W. Kim, Phys. Rev. B 61, 13115 (2000).

[35] N. S. Averkiev, L. E. Golub, and M. Willander, J. Phys.: Condens. Matter 14, R271 (2002).

[36] E. Y. Sherman, Appl. Phys. Lett. 82, 209 (2003).

[37] Y. G. Semenov, Phys. Rev. B 67, 115319 (2003).

[38] V. I. Puller, L. G. Mourokh, N. J. M. Horing, and A. Y. Smirnov, Phys. Rev. B 67, 155309 (2003).

[39] M. Glazov and E. Ivchenko, J. Supercond. 16, 735 (2003).

[40] R. Winkler, Phys. Rev. B 69, 045317 (2004).

[41] F. X. Bronold, A. Saxena, and D. L. Smith, Phys. Rev. B 70, 245210 (2004).

[42] A. A. Burkov and L. Balents, Phys. Rev. B 69, 245312 (2004).

[43] M. M. Glazov, Phys. Rev. B 70, 195314 (2004).

[44] Z. Wilamowski and W. Jantsch, Phys. Rev. B 69, 035328 (2004).

[45] D. Culcer and R. Winkler, Phys. Rev. B 76, 195204 (2007).

[46] Y. Zhou and M. W. Wu, Solid State Commun. 149, 2078 (2009).

[47] M. Murata and T. Tsuchiya, Physica E (Amsterdam, Neth.) 42, 2711 (2010).

[48] H. Akera, H. Suzuura, and Y. Egami, Phys. Rev. B 89, 075314 (2014).

[49] H. Akera, H. Suzuura, and Y. Egami, Phys. Rev. B 92, 205311 (2015).

[50] H. Akera, H. Suzuura, and Y. Egami, Phys. Rev. B 95, 045301 (2017).

[51] Y. Egami and H. Akera, Appl. Phys. Express 10, 063007 (2017).

[52] M. Huijben, G. W. J. Hassink, M. P. Stehno, Z. L. Liao, G. Rijnders, A. Brinkman, and G. Koster, Phys. Rev. B 96, 075310 (2017).

[53] E. L. Ivchenko, Sov. Phys. Solid State 15, 1048 (1973).

[54] P. Boross, B. Dóra, A. Kiss, and F. Simon, Sci. Rep. 3, 3233 (2013).

[55] G. Dresselhaus, Phys. Rev. 100, 580 (1955).

[56] N. S. Averkiev, L. E. Golub, A. S. Gurevich, V. P. Evtikhiev, V. P. Kochereshko, A. V. Platonov, A. S. Shkolnik, and Y. P. Efimov, Phys. Rev. B 74, 033305 (2006).

[57] J. Schliemann, Rev. Mod. Phys. 89, 011001 (2017).

[58] R. Lassnig, Phys. Rev. B 31, 8076 (1985). 
[59] W. Kohn and J. M. Luttinger, Phys. Rev. 108, 590 (1957).

[60] T. Ishikawa and H. Akera, Phys. Rev. B 100, 125307 (2019).

[61] A. Sasaki, S. Nonaka, Y. Kunihashi, M. Kohda, T. Bauernfeind, T. Dollinger, K. Richter, and J. Nitta, Nat. Nanotechnol. 9, 703 (2014).

[62] S. Faniel, T. Matsuura, S. Mineshige, Y. Sekine, and T. Koga, Phys. Rev. B 83, 115309 (2011).

[63] N. Tombros, S. Tanabe, A. Veligura, C. Jozsa, M. Popinciuc, H. T. Jonkman, and B. J. van Wees, Phys. Rev. Lett. 101, 046601 (2008).
[64] C. Ertler, S. Konschuh, M. Gmitra, and J. Fabian, Phys. Rev. B 80, 041405(R) (2009).

[65] D. Huertas-Hernando, F. Guinea, and A. Brataas, Phys. Rev. Lett. 103, 146801 (2009).

[66] T.-Y. Yang, J. Balakrishnan, F. Volmer, A. Avsar, M. Jaiswal, J. Samm, S. R. Ali, A. Pachoud, M. Zeng, M. Popinciuc, G. Güntherodt, B. Beschoten, and B. Özyilmaz, Phys. Rev. Lett. 107, 047206 (2011).

[67] W. Han and R. K. Kawakami, Phys. Rev. Lett. 107, 047207 (2011). 